\title{
The Inevitable Outcome of Chronic Recurring Actinomyces Osteomyelitis of The Hand
}

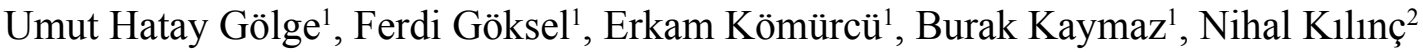 \\ ${ }^{1}$ Department of Orthopaedics and Traumatology, Çanakkale Onsekiz Mart University Faculty of Medicine, Çanakkale, Turkey \\ ${ }^{2}$ Department of Patology, Çanakkale Onsekiz Mart University Faculty of Medicine, Çanakkale, Turkey
}

A 50 year-old patient presented with pain, swelling, purulent discharge, multiple nodules, and deformity of his right hand (Figure 1). His symptoms had begun with pain and swelling without trauma 16 years ago. He had been diagnosed with chronic actinomyces osteomyelitis and underwent medical therapy and surgical debridement in four different incidents. He had been on antibiotics irregularly for 12 years. Deformity was evident, there was no active wrist movement; supination and pronation were severely restricted. Erythrocyte sedimentation rate (ESR), and C-reactive protein (CRP) levels were elevated (Sed: $120 \mathrm{~mm} / \mathrm{h}$ (0-20), CRP: $4.7 \mathrm{mg} / \mathrm{dL}$ (0-0.5)), but there was no leukocytosis. Chronic osteomyelitis of the distal forearm and hand on radiological examination. Anaerobic cultures of the pus were negative, whereas aerobic cultures yielded Staphylococcus aureus. Biopsy specimens from the nodules confirmed the previous diagnoses of chronic Actinomyces israelii. No additional diagnostic tests were necessary, and the patient underwent above elbow limb amputation. There were no complications following the surgery. A twelve month course of ampicillin/sulbactam was initiated.

Involvement of the upper extremities is very rare, and only case reports are present in the literature. So far, only 15 cases with actinomyces osteomyelitis of the hand are reported. Of these, 13 have involved metacarpals and phalanxes, and 2 have involved the carpal bones. (1-4) Our case is distinct from the literature as it is the only case with all of the bones distal to the mid-forearm involved.

Typical presentation and radiographic examination may resemble tuberculosis, fungal infections or malignancies. Anaerobic culture and biopsy are necessary for definitive diagnosis $(1,2)$. It is not always possible to isolate the microorganism. Identification of yellow sulphur granules in histological specimens is pathognomic (1-4). Mah et al. have reported

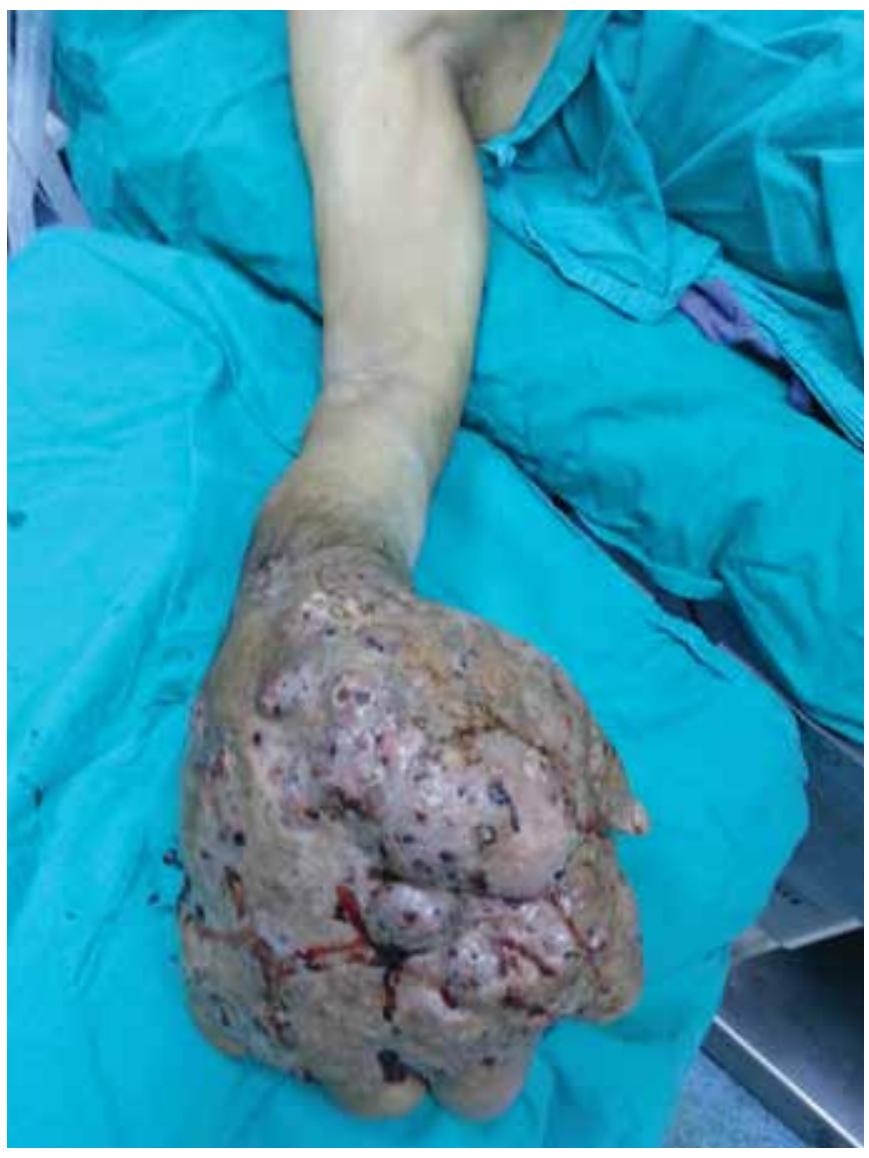

FIG. 1. Preoperative image

Actinomyces meyeri osteomyelitis in a 40 year-old patient in the fifth proximal phalanx. (2) Other cases in the literature are caused by Actinomyces israelii. In three of these cases, anaerobic cultures were negative (1-4). In our case, prolonged disease, poor patient compliance and failure to eradicate the 
pathogen resulted in a much worse clinical scenario. Carpal and metacarpal bones were completely destructed; the radius, ulna and phalanxes were severely affected by the ongoing infection. Anaerobic cultures were negative, whereas Staphylococcus aureus was isolated in aerobic cultures. Pulverer et al. reported that Staphyloccoci are usual concomitant pathogens of actinomyces. (5) Histological findings were similar to other cases in the literature and the causative agent was Actinomyces israelii.

The treatment of actinomyces osteomyelitis incudes surgical debridement combined with a 6-12 month course of large doses of penicillin, ampicillin and sulphonamides (1-4). All of the cases but two in the literature have been successfully cured with this treatment (1-4). Amputation is indicated for recurring cases, despite adequate treatment. Kundu et al. amputated the hand of a 33 year-old patient at the wrist (4). We performed above-elbow amputation in our case, which did not cure the problem, despite 4 antibiotic therapies. A 12 month course of oral ampicillin/sulbactam therapy was initiated.

Radical surgical intervention is inevitable in cases with recurring chronic actinomyces osteomyelitis. This paper presents the case with longest history of disease, largest soft tissue involvement and most radical surgical treatment.

Ethics Committee Approval: N/A.

Informed Consent: Written informed consent was obtained from patient who participated in this study.

Peer-review: Externally peer-reviewed.
Author contributions: Concept - U.H.G.; Design - F.G., B.K.; Supervision - E.K.; Resource - U.H.G., N.K.; Materials - U.H.G., N.K.; Data Collection\&/or Processing - U.H.G., N.K., F.G.; Analysis\&/or Interpretation - F.G., B.K.; Literature Search - F.G., U.H.G.; Writing - F.G.; Critical Reviews - E.K., F.G.

Conflict of Interest: No conflict of interest was declared by the authors.

Financial Disclosure: The authors declared that this study has received no financial support.

\section{REFERENCES}

1. Mert A, Bilir M, Bahar H, Torun M, Tabak F, Ozturk R, et al. Primary actinomycosis of the hand: a case report and literature review. Int J Infect Dis 2001;5:112-4. [CrossRef]

2. Mah E, Stanley P, McCombe DB. Actinomycosis infection of the finger. Hand Surg 2005;10:285-8. [CrossRef]

3. Kargi E, Akduman D, Gungor E, Deren O, Albayrak L,Erdogan B. Primay extremity actinomycosis causing osteomyelitis of the hand. Plast Reconstr Surg 2003:112:1495-7. [CrossRef]

4. Kundu ZS, Singh R, Rana P. Actinomycosis Of Hand And Wrist: A Case Report. The Internet Journal of Orthopedic Surgery 2007. Volume 5 Number 1.

5. Pulverer G1, Schütt-Gerowitt H, Schaal KP. Human cervicofacial actinomycoses: microbiological data for 1997 cases. Clin Infect Dis 2003;37:490-7. [CrossRef] 